\title{
Inhomogeneous response of an ion ensemble from mechanical stress
}

\author{
S. Zhang, ${ }^{1}$ N. Galland, ${ }^{1,2}$ N. Lučić, ${ }^{1}$ R. Le Targat $\odot,{ }^{1}$ A. Ferrier, ${ }^{3,4}$ P. Goldner, ${ }^{3}$ B. Fang, ${ }^{1}$ Y. Le Coq, ${ }^{1}$ and S. Seidelin $\odot^{2,5,}$ \\ ${ }^{1}$ LNE-SYRTE, Observatoire de Paris, Université PSL, CNRS, Sorbonne Université, Paris, France \\ ${ }^{2}$ Univ. Grenoble Alpes, CNRS, Grenoble INP, and Institut Néel, 38000 Grenoble, France \\ ${ }^{3}$ Chimie ParisTech, Université PSL, CNRS, Institut de Recherche de Chimie Paris, 75005 Paris, France \\ ${ }^{4}$ Faculté des Sciences et Ingénierie, Sorbonne Université, UFR No. 933, 75005 Paris, France \\ ${ }^{5}$ Institut Universitaire de France, 103 Boulevard Saint-Michel, 75005 Paris, France
}

(Received 24 December 2019; accepted 20 February 2020; published 12 March 2020)

\begin{abstract}
Material strain has recently received growing attention as a complementary resource to control the energy levels of quantum emitters embedded inside a solid-state environment. Some rare-earth ion dopants provide an optical transition which simultaneously has a narrow linewidth and is highly sensitive to strain. In such systems, the technique of spectral hole burning, in which a transparent window is burned within the large inhomogeneous profile, allows one to benefit from the narrow features, which are also sensitive to strain, while working with large ensembles of ions. However, working with ensembles may give rise to inhomogeneous responses among different ions. We investigate experimentally how the shape of a narrow spectral hole is modified due to external mechanical strain, in particular, the hole broadening as a function of the geometry of the crystal sites and the crystalline axis along which the stress is applied. Studying these effects is essential in order to optimize the existing applications of rare-earth-doped crystals in fields which already profit from the more-well-established coherence properties of these dopants, such as frequency metrology and quantum information processing, or even suggest alternative applications of these materials, for example, as robust devices for force-sensing or highly sensitive accelerometers.
\end{abstract}

DOI: 10.1103/PhysRevResearch.2.013306

The extraordinary coherence properties of rare-earth ion dopants in a host crystal have led to their description in terms of a "frozen ion gas" as they constitute the solid-state emitters that come the closest to isolated ions trapped with electromagnetic fields inside a vacuum chamber in terms of coherence properties [1-4]. However, the impact of the crystalline matrix cannot be entirely neglected, as illustrated, for instance, by the large inhomogeneous absorption profile of the ion ensemble, arising from a nonuniform distribution of static strain due to the doping which slightly distorts the crystalline lattice locally. The technique of spectral hole burning has been shown to surmount such inhomogeneous effects, as ions with a particular frequency are selectively pumped to a hyperfine dark state. The width of the created spectral hole, despite the fact that a large number of ions participate in the signal, reflects the single-ion characteristics, and as long as the crystal is not subject to external perturbations, these characteristics are preserved in time. This has been extensively exploited, for instance, in ultrahigh-precision laser stabilization and spectroscopy [5-7], in which a laser is frequency stabilized by locking it to a narrow spectral hole. However, if the crystal containing a spectral hole is subject to modifications in its

\footnotetext{
*signe.seidelin@neel.cnrs.fr

Published by the American Physical Society under the terms of the Creative Commons Attribution 4.0 International license. Further distribution of this work must maintain attribution to the author(s) and the published article's title, journal citation, and DOI.
}

physical environment (for instance, external stress), the ions constituting the spectral hole may react differently according to their local environment, and in addition to a global displacement of the spectral hole, a deformation can occur. In order to fully benefit from solid-state systems based on ensembles, such effects must be studied and understood.

In this work we are particularly interested in the 580-nm optical transition in $\mathrm{Eu}^{3+}$ ions in an $\mathrm{Y}_{2} \mathrm{SiO}_{5}$ (YSO) host matrix, as it has an ultranarrow linewidth, potentially down to $122 \mathrm{~Hz}$ [2], and is sensitive to mechanical strain, allowing for an efficient strain coupling between dopants and the crystalline matrix. Earlier experiments have demonstrated such strain coupling in the context of optomechanical systems, for instance, using quantum dots [8] or nitrogen vacancy (NV) centers in diamond [9-11], but these emitters exhibited either a large linewidth or low strain sensitivity. Generally speaking, optical transitions exhibit relatively high strain sensitivity, but in most solid-state emitters, such transitions exhibit very broad (in the gigahertz range) linewidths with, at best, zerophonon lines in the megahertz range. On the contrary, the electronic structure of trivalent lanthanide ions, in which the optical transitions inside the $4 f$ shell are protected by $5 s$ and $5 p$ fully populated shells, allows for record narrow transitions as well as the high strain sensitivity that is typical for an optical transition. Thus, when viewing the strain sensitivity as a resource, this kind of system represents the best of both worlds, as witnessed by proposals for creating strainengineered quantum hybrid systems based on this system $[12,13]$. On the other hand, depending on the application, this high sensitivity can also be a nuisance, of which the effect 
(a)

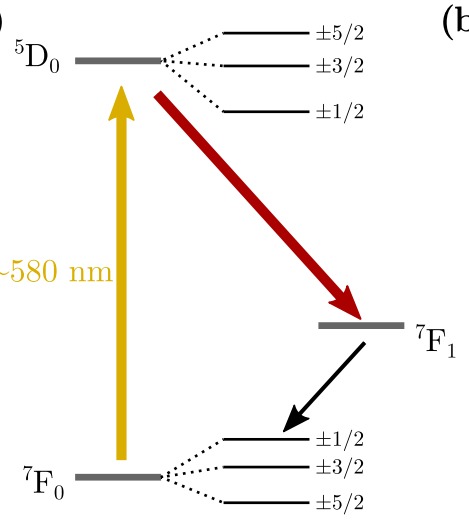

(b)

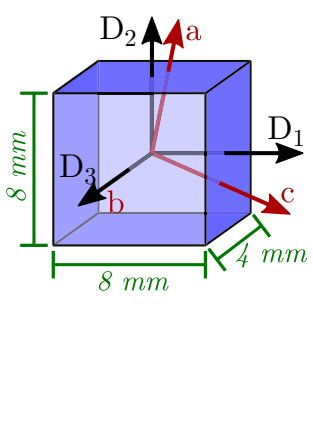

FIG. 1. Details of the ion level structure and crystal axes. (a) Energy diagram of the $\mathrm{Eu}^{3+}$ ions, where each electronic level is separated into three hyperfine states. When excited at resonance at $580 \mathrm{~nm}$, the ions decay radiatively primarily to ${ }^{7} F_{1}$ and then nonradiatively to one of the hyperfine states. (b) $\mathrm{The}_{2} \mathrm{Y}_{2} \mathrm{SiO}_{5}$ crystal, cut parallel to the three dielectric axes $D_{1}, D_{2}$, and $D_{3}$, as well as its dimensions.

needs to be minimized. For instance, vibrations of the sample give rise to an acceleration of the crystal which leads to a compression, in turn creating fluctuations in strain [14], which can limit the performance of the system. Such vibrations often occur due to the operation of a cryostat and represent a recurring challenge for many applications with this type of dopant. Therefore, whether a resource or a nuisance, an in depth study of the response of $\mathrm{Eu}^{3+}$ in an YSO matrix to strain seems valuable in a variety of contexts.

We study the ${ }^{7} F_{0} \rightarrow{ }^{5} D_{0}$ transition shown in Fig. 1(a). More precisely, the YSO crystal possesses two locations within the unit cell where $\mathrm{Eu}^{3+}$ can substitute for $\mathrm{Y}^{3+}$, referred to as sites 1 and 2, with vacuum wavelengths of 580.04 and $580.21 \mathrm{~nm}$, respectively. These are the only two nonequivalent substitution sites in the crystalline matrix, both exhibiting a $C_{1}$ symmetry. Each state is composed of three hyperfine states, with mutual separations in the $30-100 \mathrm{MHz}$ range [1]. As illustrated in Fig. 1(a), the spectral holes are formed by pumping the ions resonantly from the ${ }^{7} F_{0}$ to the ${ }^{5} D_{0}$ state. From there, they decay radiatively, mainly to the ${ }^{7} F_{1}$ state [15], before decaying nonradiatively to the three hyperfine states in the ${ }^{7} F_{0}$ manifold. Optical pumping prevents population buildup in the hyperfine level with which the pump beam is resonant, creating a transparent window in the inhomogeneous profile at this exact frequency. A spectral hole represents approximately $10^{13} \mathrm{Eu}^{3+}$ ions.

We recently determined the frequency shift of this transition by studying the linear displacement in frequency of a spectral hole when applying external stress to the crystal [16]. Here we will instead focus on how the shape of the spectral hole evolves according to the applied stress, for these two different crystal sites, as this provides insight into the microscopic environment of the ion dopants constituting the spectral hole. As the direction parallel to which the stress is applied also plays an important role in the overall frequency shift as well as in the broadening, we investigate the application of stress along two nonequivalent axes: parallel to either the $D_{1}$ or the $D_{2}$ dielectric axis [see Fig. 1(b)]. For (a)

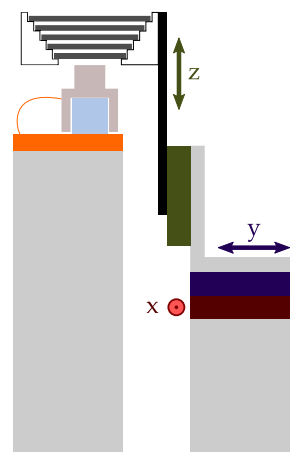

(b)

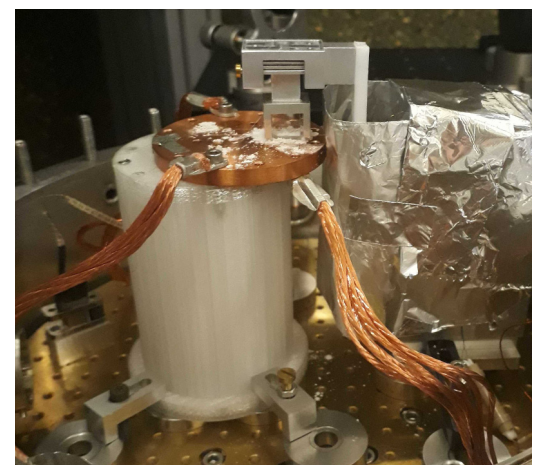

FIG. 2. Experimental device for applying a calibrated stress to the crystal. (a) Schematic of the ladder structure which allows us to deposit a given number of weights on top of the crystal (illustrated by the light blue square), by vertically displacing the structure ( $z$-axis translation stage), and to center the weights (by using the $x-y$ axis). (b) Photograph of the device, mounted inside the cryostat science chamber. In both panels an electrically grounded metal structure surrounding the crystal is visible, which prevents a buildup of static charges.

maximum absorption, the pump beam is propagated along the crystalline $b$ axis (which coincides with the dielectric $D_{3}$ axis) and polarized linearly along the $D_{1}$ axis $[15,17]$.

The physical setup is similar to the one described in detail elsewhere [7,16]. In brief, we use two diode lasers (master and slave) at $1160 \mathrm{~nm}$. The master laser is locked to a frequency stabilization cavity by the Pound-Drever-Hall method allowing for a frequency instability below $10^{-14}$ for time constants of 1-100 s (corresponding to a linewidth of a few hertz). The slave laser is locked to the master laser. They are both subsequently frequency doubled to reach 580 $\mathrm{nm}$, with an output intensity of approximately $5 \mathrm{~mW}$. The slave laser possesses the same stability as the master, but its frequency is continuously tunable in a range of several gigahertz. In addition, acousto-optic modulators allow us to scan the frequency across the spectral structures with a range of approximately $1 \mathrm{MHz}$. An absolute frequency measurement is provided by referencing the signal to a frequency comb. To monitor the shape of the spectral hole, we use an avalanche photodiode to record the laser intensity after absorption of the crystal, normalized by the laser intensity send directly to the detector. Polarizing beam splitters combined with $\lambda / 2$ waveplates allow for adjusting the intensity. The crystal is maintained at $3.15 \mathrm{~K}$ in a closed-loop cryostat positioned on an active vibration isolation platform.

In order to quantitatively study the response of a spectral structure to mechanical stress, a calibrated force in a given direction must be applied at cryogenic temperature. We use a staircaselike structure [see Fig. 2(a)], with a vertical position being controlled by a cryocompatible motorized platform, providing a way of placing a variable number of weights on the top of the crystal inside the science chamber in the cryostat [see Fig. 2(b)]. From the mass of the weights, we have access to an absolute calibrated force. We start the sequence by burning a spectral hole with no weights on the crystal and we 


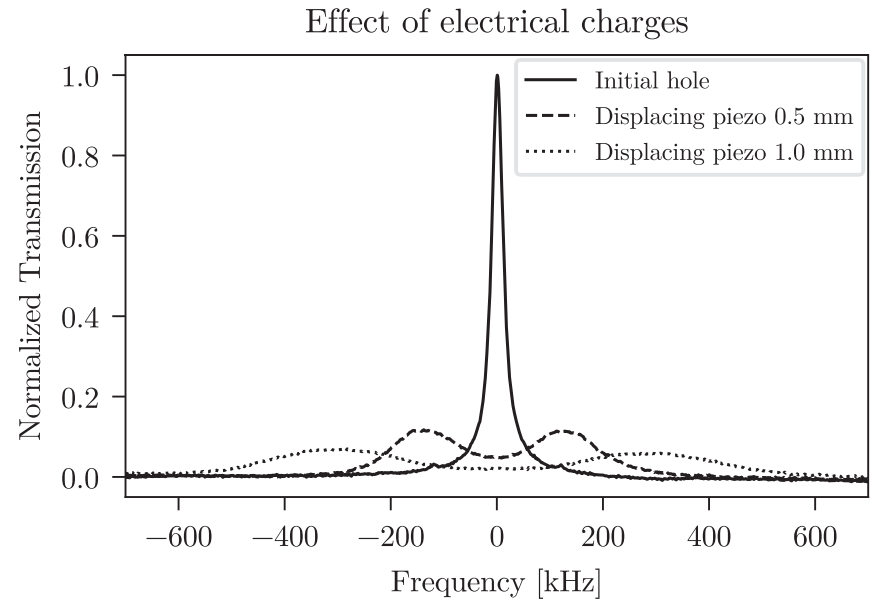

FIG. 3. Effect on a spectral hole of operating the piezoelectric translation stage near the crystal, for crystal site 1 (without applying weights) prior to electrical shielding. The solid line corresponds to the initial spectral hole and the dashed and dotted lines to the hole after having operated the piezoelectric translation stage one and two steps, respectively. The deformations of the spectral holes persist in time, despite the operation of the piezoelectric translation stage having ended.

then add the number of weights corresponding to the desired stress.

During the initial stages of the experiment, we observed irreversible effects on the spectral hole while operating the translation stage for depositing the weights. More precisely, by operating the piezoelectric translation stage, even without depositing weights on the crystal, the hole would exhibit a splitting and moreover the spectral hole would not return to its initial shape, even after the piezoelectric operation has ended (note, however, that no permanent microstructural modification of the crystal was observed). Examples of this behavior are illustrated in Fig. 3, where we show an example of the shape of a spectral hole after having operated the piezoelectric stage for a given excursion distance. This behavior can be understood as the energy transitions in $\mathrm{Eu}^{3+}$ are also sensitive to electric fields [18-20], and when operating the motorized translation stage (which works by the stick-slip mechanism of a piezo crystal on a fine threaded screw), accumulated stray charges can generate irreversible displacements or deterioration of the spectral hole. Moreover, silver lacquer on the crystal used for optimal thermal contact seemed to exert an additional stress on the crystal while applying weights, modifying the spectral hole in a manner depending of the amount and position of the silver lacquer in contact with the crystal. The solution we adopted consists in removing the silver lacquer and replacing it with a metal shield positioned on the top of, and extending down around, the crystal, but without direct contact with the vertical sides of the crystal. In addition, we surrounded the entire piezoelectric translation stage with a metal cover [visible in Fig. 2(b)]. Both the crystal and piezoelectric stage cover were subsequently grounded. The weights for generating stress, instead of being deposited directly on the crystal, were then put on the crystal metal shield. These modifications resulted in the disappearance of

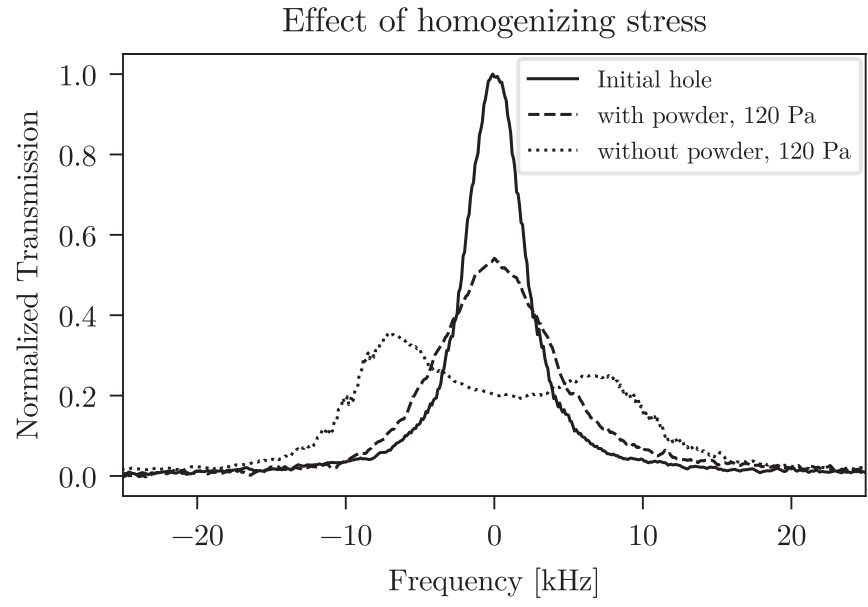

FIG. 4. Effect of stress on the shape of the spectral hole, for crystal site 2 , with stress applied along the $D_{1}$ axis. The solid line shows the initial hole without stress. The dotted line corresponds to the case in which the stress is applied without depositing a crystalline powder at the contact surfaces and the dashed line to the case in which a powder has been used. The stress also gives rise to a global shift in frequency of the spectral structures, but this shift has been subtracted in order to center the curves in frequency.

any effects on shape of the spectral hole due to operating the piezoelectric translation stage.

However, when subsequently adding weights to the crystal, instead of a pure shift in frequency of the spectral hole, we observed a clear splitting in the spectra. As such splitting is not expected to appear as long as the stress is applied perpendicular to the $b$ axis [21] (which is our case, as we apply stress parallel to either $D_{1}$ or $D_{2}$ ), we suspected that the stress was not perfectly parallel to the axis chosen. One potential explanation was that the weights were not completely centered on the crystal. To improve on this aspect, an $x-y$ translation stage was added for centering the objects on the crystal, and the splitting seemed to decrease slightly, but was not eliminated. We suspected that the contact surface between the weights and crystal, despite all the care taken, was not sufficiently uniform as to create a strain field perfectly perpendicular to the $b$ axis inside the crystal. In order to homogenize the stress, we used an approximately 1-mm layer of YSO powder with a grain size of the order of micrometers at the two contact surfaces of the crystal. That is, we deposited the powder on top of the crystal (between the crystal and the metal shield) and below the crystal (between the crystal and the cryostat platform). When repeating the experiment, we observed that the splitting was no longer visible for the given pressures. These effects are illustrated in Fig. 4, in which we have applied stress parallel to the $D_{1}$ axis. The solid line corresponds to the initial spectral hole with a width below $5 \mathrm{kHz}$. When applying stress, before homogenizing the stress with the crystalline powder, we obtained spectra exhibiting large splittings, as illustrated by the dotted line, which appear to be eliminated by the use of the crystal powder (dashed line).

We do observe, however, a persistent broadening of the spectral hole with applied stress, an effect we wish to investigate quantitatively in the following. First, in order to 
Spectral hole broadening

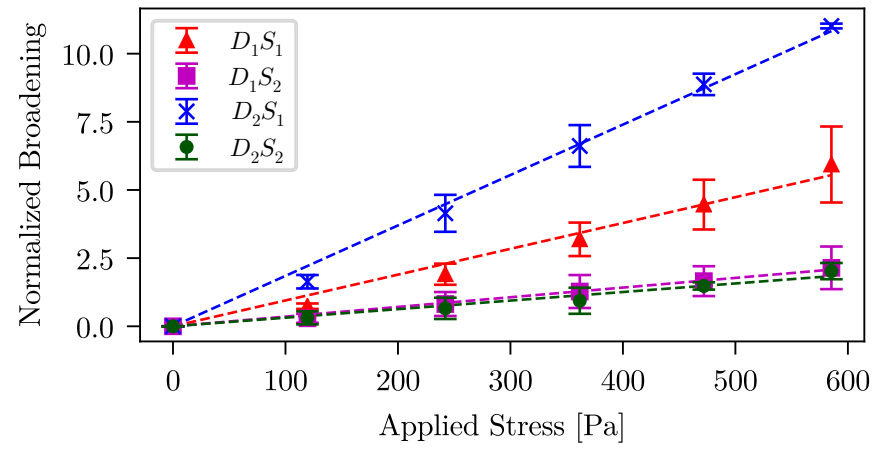

FIG. 5. Effect of the stress on the width of a spectral hole for different directions parallel to which the stress is applied $\left(D_{1}\right.$ and $\left.D_{2}\right)$ and crystal sites $\left(S_{1}\right.$ and $\left.S_{2}\right)$. The broadening has been normalized by the strain sensitivity in order to plot $\left(\Gamma-\Gamma_{0}\right) /\left(\Gamma_{0}\left|\kappa_{D_{i} S_{j}}\right| / \kappa_{D_{1} S_{1}}\right)$, where $\Gamma_{0}$ is the initial width of the unperturbed hole. The dashed lines are linear fits to the data.

verify that the strain does not exhibit a significant variation across the crystal, after burning a spectral hole and adding strain, we probe the spectral hole in five different locations (center, left, right, top, and bottom) of the crystal, with a beam diameter of $2 \mathrm{~mm}$. The spectral hole appears to have the same shape in the different location, and the same global shift in frequency (with a variation much smaller than the width of the spectral hole), eliminating the possibility of a strong strain gradient across the crystal. In order to study the residual broadening systematically, we record the shape of the spectral hole as a function of stress applied (corresponding to one to five weights added to the crystal) with a $2.5-\mathrm{mm}$ beam diameter, centered in the middle of the crystal. The experiment is repeated for both crystal sites $\left(S_{1}\right.$ and $\left.S_{2}\right)$ and for adding the stresses parallel to the $D_{1}$ and $D_{2}$ axes. The result of this study is shown in Fig. 5. By denoting the initial width of the spectral hole, before applying stress, by $\Gamma_{0}$ and the width measured for a given stress by $\Gamma$, the broadening is given by $\left(\Gamma-\Gamma_{0}\right) / \Gamma_{0}$. For the data in Fig. 5 , the initial hole widths are the following: $\Gamma_{0}\left(D_{1} S_{1}\right)=5.4 \mathrm{kHz}, \Gamma_{0}\left(D_{1} S_{2}\right)=4.0 \mathrm{kHz}$, $\Gamma_{0}\left(D_{2} S_{1}\right)=3.8 \mathrm{kHz}$, and $\Gamma_{0}\left(D_{2} S_{2}\right)=4.4 \mathrm{kHz}$. We checked that the width of the initial hole does not change (to within the uncertainty of the determination of the width of the hole) in the temperature range between 3.1 and $4.2 \mathrm{~K}$. As our actual temperature fluctuations are at the $1-\mathrm{mK}$ level, the width of the initial hole should therefore remain constant during the measurement sequence. This is further confirmed by removing all weights at the end of the measurement sequence and observing that the hole regains its initial shape.

Moreover, in the case that the broadening arises from a residual inhomogeneity in the amplitude of the stress field in the crystal across the optical spot, the broadening should depend directly on the strain sensitivity, which is different for different crystal sites and axes parallel to which the stress is applied. That is, the same gradient in strain should give rise to a larger broadening for a more sensitive site and direction of stress. Thus, in order to compare the different measurements, we have also normalized the broadening according to the sensitivity. We previously determined these values
[16], $\kappa_{D_{1} S_{1}}=46 \pm 17 \mathrm{~Hz} \mathrm{P^{-1 }}, \kappa_{D_{1} S_{2}}=137 \pm 16 \mathrm{~Hz} \mathrm{~Pa}^{-1}$, $\kappa_{D_{2} S_{1}}=-19 \pm 10 \mathrm{~Hz} \mathrm{~Pa}^{-1}$, and $\kappa_{D_{2} S_{2}}=-213 \pm 13 \mathrm{~Hz} \mathrm{~Pa}^{-1}$, where we have used the notation $\kappa_{D_{i}} S_{j}$, where $D_{i}$ corresponds to the direction parallel to which the strain is applied and $S_{j}$ to the crystal site $(i, j=1$ or 2$)$. Using these values, we choose to normalize the broadening to the sensitivity of the crystal site 1 , with a stress applied parallel to the $D_{1}$ axis. That is, we plot $\left(\Gamma-\Gamma_{0}\right) /\left(\Gamma_{0}\left|\kappa_{D_{i} S_{j}}\right| / \kappa_{D_{1} S_{1}}\right)$. The results are shown in Fig. 5. Here the error bars account for the reproducibility of the experiment (four to six measurements per data point) and solid lines correspond to linear fits to the data. By allowing for a quadratic component, we notice a small improvement of the quality of the fit, but the linear contribution is clearly dominant, so in what follows, we will consider this component only.

Had the broadening simply been due uniquely to an inhomogeneity in the amplitude of the crystal strain, then we would expect all linear fits to exhibit to within the error bars, as we have normalized the broadening to the strain sensitivity. However, we observe a significant variations in slopes: $\alpha_{D_{1} S_{1}}=9.5 \pm 4.6 \mathrm{kPa}^{-1}, \alpha_{D_{1} S_{2}}=3.6 \pm 2.1 \mathrm{kPa}^{-1}$, $\alpha_{D_{2} S_{1}}=18.5 \pm 0.2 \mathrm{kPa}^{-1}$, and $\alpha_{D_{2} S_{2}}=3.1 \pm 0.1 \mathrm{kPa}^{-1}$. The uncertainties on the slopes account for the repeatability of the experiment. Even taking into account the uncertainty on both the $\kappa_{D_{i} S_{j}}$ and the $\alpha_{D_{i} S_{j}}$ coefficients, $\alpha_{D_{2} S_{1}}$ cannot be brought to overlap with $\alpha_{D_{1} S_{2}}$ and $\alpha_{D_{2} S_{2}}$. We cannot exclude a small inhomogeneity along the axis of propagation of the probe beam (along the $b$ axis). However, a gradient in the amplitude of strain in this direction would also give rise to a broadening being identical (when normalized by the sensitivity) among sites and stress directions. In order to explain the variations of the slopes due to the inhomogeneity in the strain, one would require a component of the force to be in the direction perpendicular to the applied stress direction. This is thus unlikely to account for the differences observed.

Instead, we may, at least partially, attribute the observed effect to an inhomogeneity of the different ions' responses on a microscopic scale, as suggested by other authors for a different system [22]. In our case, the ions forming the spectral hole all have a slightly different electromagnetic environment, coming from a nonuniform distribution of static strain within the crystal which stems essentially from local distortion of the crystal lattice due to doping, which also accounts for the large inhomogeneous profile. Although the ions contributing to the spectral hole all have the same absorption frequency (to within the width of the hole), this frequency is both a combination of the exact hyperfine states between which they have transitioned, which are separated by several tens of megahertz (all three hyperfine levels are populated at $4 \mathrm{~K}$ prior to optical pumping), and the local static crystal strain. That is, contributing to a single spectral hole, there will be ions with internal static strain varying in both amplitude and direction and they are therefore likely to react differently according to the externally applied strain. Although the strain sensitivities cited above have been shown to be independent of the frequency position in the inhomogeneous profile [16], this explanation can still be valid: In each position of the profile, a spectral hole will be formed by ions with a different local strain environment (reacting differently to strain), but the global strain sensitivity corresponds to an 
average effect of the ensemble of the ions, which can still be identical to that of a second position in the inhomogeneous profile.

Finally, another type of inhomogeneity on a microscopic level could stem from the fact that transitions among different hyperfine states could react differently to strain as a direct consequence of atomic structure, but comparing with other systems, we believe the corresponding variation may be negligible. For instance, for the $\mathrm{NV}^{-}$in diamond, the strain sensitivity of the optical 638-nm transition is $1000 \mathrm{~Hz} \mathrm{\textrm {Pa } ^ { - 1 }}$ [23], while for the ground-state spin levels, the sensitivity only amounts to $0.01 \mathrm{~Hz} \mathrm{~Pa}^{-1}$ [10], thus $10^{5}$ times smaller. Of course, dedicated measurements will be required in order to confirm whether this also applies to the case of rare-earth ion dopants, but the absence of distinct components in the profile of the spectral hole subject to stress also supports this assumption.

In conclusion, we have discussed how the shape of a narrow spectral hole is sensitive to stray charges and we have shown how to eliminate these detrimental effects. We have also demonstrated that adding a fine layer of crystalline powder at the contact interfaces of a crystal significantly reduces inhomogeneities, in both direction and amplitude, in the strain field generated in the crystal due to added weights. This is a prerequisite for studying the inhomogeneities of the ion dopants on a microscopic level. The fact that the broadening, normalized by the strain sensitivity, is distinct for different crystal sites and directions for the stress indicates that the various ions participating in the spectral structure react differently according to their local strain environment. Future work should include repeating the experiments with a much smaller number of ions to see how this influences the inhomogeneous effects. Ideally, one would study the response to strain of a single ion, but currently, the smallest number of $\mathrm{Eu}^{3+}$ ions in an YSO matrix detected is approximately 10 [24], made possible by enhancing the signal by means of a microcavity, and thus currently incompatible with the application of an external calibrated stress. However, by using a protocol of hyperfine class selection already demonstrated for rare-earth ion dopants [25] it should be possible to assess to what extent different classes react differently to strain. Theoretical calculations of the frequency shift of an ion dopant as a function of its local strain environment would also be of great value to better understand inhomogeneous effects resulting in spectral hole broadening, but due to the lack of symmetry of the crystal and the substitution sites, such calculations are extremely challenging. Even if the exact positions of the individual atoms can be calculated under strain, the calculation of $\mathrm{Eu}^{3+}$ energy levels under strain would still have to be performed. This is currently out of reach of the best crystal field models. At present, it is therefore not possible to propose a model describing the results; thus the measurements seem to be the most efficient avenue for investigating these effects.

This work was supported by the European Union's Horizon 2020 research and innovation program under Grant Agreement No. 712721 (NanOQTech). It also received support from the Ville de Paris Emergence Program, the Région Ile de France DIM C'nano and SIRTEQ; the LABEX Cluster of Excellence FIRST-TF (Grant No. ANR-10-LABX-48-01) within the program "Investissements d'Avenir" operated by the French National Research Agency; the EMPIR 15SIB03 OC18 from the EMPIR program co-financed by the Participating States and from the European Union's Horizon 2020 research and innovation program.
[1] R. Yano, M. Mitsunaga, and N. Uesugi, Ultralong optical dephasing time in $\mathrm{Eu}^{3+}: \mathrm{Y}_{2} \mathrm{SiO}_{5}$, Opt. Lett. 16, 1884 (1991).

[2] R. W. Equall, Y. Sun, R. L. Cone, and R. M. Macfarlane, Ultraslow Optical Dephasing in $\mathrm{Eu}^{3+}: \mathrm{Y}_{2} \mathrm{SiO}_{5}$, Phys. Rev. Lett. 72, 2179 (1994).

[3] C. W. Thiel, T. Böttger, and R. L. Cone, Rare-earth-doped materials for applications in quantum information storage and signal processing, J. Lumin. 131, 353 (2011).

[4] P. Goldner, A. Ferrier, and O. Guillot-Noël, in Handbook on the Physics and Chemistry of Rare Earths, edited by J.-C. G. Bünzli and V. K. Pecharsky (Elsevier, Amsterdam, 2015), Vol. 46, pp. 1-78.

[5] B. Julsgaard, A. Walther, S. Kröll, and L. Rippe, Understanding laser stabilization using spectral hole burning, Opt. Express 15, 11444 (2007).

[6] M. J. Thorpe, L. Rippe, T. M. Fortier, M. S. Kirchner, and T. Rosenband, Frequency stabilization to $6 \times 10^{-16}$ via spectralhole burning, Nat. Photon. 5, 688 (2011).

[7] O. Gobron, K. Jung, N. Galland, K. Predehl, R. Letargat, A. Ferrier, P. Goldner, S. Seidelin, and Y. Le Coq, Dispersive heterodyne probing method for laser frequency stabilization based on spectral hole burning in rare-earth doped crystals, Opt. Express 25, 15539 (2017).
[8] I. Yeo, P.-L. de Assis, A. Gloppe, E. Dupont-Ferrier, P. Verlot, N. S. Malik, E. Dupuy, J. Claudon, J.-M. Gérard, A. Auffèves, G. Nogues, S. Seidelin, J. Poizat, O. Arcizet, and M. Richard, Strain-mediated coupling in a quantum dot-mechanical oscillator hybrid system, Nat. Nanotechnol. 9, 106 (2014).

[9] J. Teissier, A. Barfuss, P. Appel, E. Neu, and P. Maletinsky, Strain Coupling of a Nitrogen-Vacancy Center Spin to a Diamond Mechanical Oscillator, Phys. Rev. Lett. 113, 020503 (2014).

[10] P. Ovartchaiyapong, K. W. Lee, B. A. Myers, and A. C. Bleszynski Jayich, Dynamic strain-mediated coupling of a single diamond spin to a mechanical resonator, Nat. Commun. 5, 4429 (2014).

[11] E. R. MacQuarrie, M. Otten, S. K. Gray, and G. D. Fuchs, Cooling a mechanical resonator with nitrogen-vacancy centres using a room temperature excited state spin-strain interaction, Nat. Commun. 8, 14358 (2017).

[12] K. Mølmer, Y. Le Coq, and S. Seidelin, Dispersive coupling between light and a rare-earth-ion-doped mechanical resonator, Phys. Rev. A 94, 053804 (2016).

[13] S. Seidelin, Y. Le Coq, and K. Mølmer, Rapid cooling of a strain-coupled oscillator by an optical phase-shift measurement, Phys. Rev. A 100, 013828 (2019). 
[14] A. Louchet-Chauvet, R. Ahlefeldt, and T. Chanelière, Piezospectroscopic measurement of high-frequency vibrations in a pulse-tube cryostat, Rev. Sci. Instrum. 90, 034901 (2019).

[15] F. Könz, Y. Sun, C. W. Thiel, R. L. Cone, R. W. Equall, R. L. Hutcheson, and R. M. Macfarlane, Temperature and concentration dependence of optical dephasing, spectral-hole lifetime, and anisotropic absorption in $\mathrm{Eu}^{3+}: \mathrm{Y}_{2} \mathrm{SiO}_{5}$, Phys. Rev. B 68 , 085109 (2003).

[16] N. Galland, N. Lučić, B. Fang, S. Zhang, R. Letargat, A. Ferrier, P. Goldner, S. Seidelin, and Y. Le Coq, Mechanical tunability of an ultra-narrow spectral feature with uniaxial stress, arXiv:1912.10885.

[17] A. Ferrier, B. Tumino, and P. Goldner, Variations in the oscillator strength of the ${ }^{7} F_{0}-{ }^{5} D_{0}$ transition in single crystals, J. Lumin. 170, 406 (2016).

[18] M. J. Thorpe, D. R. Leibrandt, and T. Rosenband, Shifts of optical frequency references based on spectral-hole burning in $\mathrm{Eu}^{3+}: \mathrm{Y}_{2} \mathrm{SiO}_{5}$, New J. Phys. 15, 033006 (2013).

[19] R. M. Macfarlane, A. Arcangeli, A. Ferrier, and P. Goldner, Optical Measurement of the Effect of Electric Fields on the
Nuclear Spin Coherence of Rare-Earth Ions in Solids, Phys. Rev. Lett. 113, 157603 (2014).

[20] Q. Li, Y. Bao, A. Thuresson, A. N. Nilsson, L. Rippe, and S. Kröll, Slow-light-based optical frequency shifter, Phys. Rev. A 93, 043832 (2016).

[21] J. G. Bartholomew, Investigation of the scalability of rareearth-ion quantum hardware, Ph.D. thesis, Australian National University, 2014.

[22] R. J. Reeves and R. M. Macfarlane, Measurement of the effect of uniaxial stress on the spectrum of $\mathrm{CaF}_{2}: \mathrm{Pr}^{3+}: \mathrm{D}^{-}$using spectral hole burning, Phys. Rev. B 39, 5771 (1989).

[23] G. Davies and M. F. Hamer, Optical studies of the $1.945 \mathrm{eV}$ vibronic band in diamond, Proc. R. Soc. Lond. A 348, 285 (1976).

[24] B. Casabone, J. Benedikter, T. Hümmer, F. Oehl, K. de Oliveira Lima, T. W. Hänsch, A. Ferrier, P. Goldner, H. de Riedmatten, and D. Hunger, Cavity-enhanced spectroscopy of a few-ion ensemble in $\mathrm{Eu}^{3+}: \mathrm{Y}_{2} \mathrm{O}_{3}$, New J. Phys. 20, 095006 (2018).

[25] B. Lauritzen, N. Timoney, N. Gisin, M. Afzelius, H. de Riedmatten, Y. Sun, R. M. Macfarlane, and R. L. Cone, Spectroscopic investigations of $\mathrm{Eu}^{3+}: \mathrm{Y}_{2} \mathrm{SiO}_{5}$ for quantum memory applications, Phys. Rev. B 85, 115111 (2012). 